\title{
Let's Prevent Diabetes: From idea to implementation
}

\author{
Laura J Gray ${ }^{1}$, Jacqui Troughton ${ }^{2}$, Kamlesh Khunti ${ }^{3}$, Melanie J Davies ${ }^{3}$ \\ ${ }^{1}$ Department of Health Sciences, University of Leicester \\ ${ }^{2}$ Leicester Diabetes Centre, University Hospital of Leicester \\ ${ }^{3}$ Diabetes Research Centre, University of Leicester
}

\begin{abstract}
The prevention of Type 2 Diabetes Mellitus (T2DM) is a global health care priority. We describe a programme of work, which developed a method for identifying those with Non Diabetic Hyperglycaemia (NDH) (a high risk group in which T2DM is preventable) in primary care and developed and tested a prevention programme suitable for delivery within the NHS. The subsequent implementation of this programme as part of the NHS Diabetes Prevention Programme, which was launched in 2016, is then described.
\end{abstract}

\section{Introduction}

The prevention of Type 2 Diabetes Mellitus (T2DM) is a global health care priority and was highlighted as a national priority in the NHS 'Five Year Forward View' which outlined the NHS's ambition to become the first country to implement at scale an evidence-based T2DM prevention programme (1). T2DM is preceded by a high risk state called NDH, also known as prediabetes, where glucose levels are elevated but do not cross the diagnostic threshold for T2DM. In England, 5 million people are estimated to have NDH (2), a number which is expected to increase over the coming decades.

Once identified with NDH, there is robust evidence that T2DM can be prevented or delayed. Pivotal trials conducted globally have shown that lifestyle modification programmes that promote a healthy diet, weight loss, and increased physical activity could reduce the incidence of T2DM by up to 58\% and are at least if not more effective than pharmacological intervention (3). These programmes were very resource intensive (for example the USA programme included 16 one-hour one-to-one counselling sessions followed by an average of eight additional contacts and two telephone consultations) (4); thus it has been challenging to replicate these findings in programmes suitable for mass delivery within settings such as the NHS (5).

\section{Development and validation of a risk score for identifying those at risk}

NICE recommend a two-stage approach for identifying those with NDH - using a non-invasive risk score followed by a blood test in those with a high risk score (6). This method has been shown to be cost saving compared to using a blood test alone (7). Risk scores calculate an individual's risk of having or developing a condition based on their risk factors. Risk scores not only target blood testing, they also engage individuals with their risk factors and help to explain why someone is at high risk in a way a result from a blood test cannot. Initially we developed a risk score which was designed to be completed by members of the public to see if they were at risk of having undiagnosed NDH or T2DM (8). This score is calculated by assessing age, sex, ethnicity, body mass index, waist circumference, family history of diabetes and high blood pressure. This score is available at https://riskscore.diabetes.org.uk/.

As part of this programme we wanted to adapt this score for use in primary care. We wanted to develop and validate a score which could be calculated using data stored in primary care medical records. This score would be able to identify all those at risk of NDH within a practice who could then be invited for screening. The score was adapted in a number of ways: (1) waist circumference was removed as this is not well recorded in primary care; (2) we used a more sophisticated scoring 
method as this would be calculated by a piece of software; (3) we developed software to run the score in practice and output a spreadsheet of those at risk to be used for inviting people for screening. This score was developed using data from the ADDITION-Leicester study and validated using data from the $\operatorname{STAR}$ study $(9,10)$. It was shown to have acceptable levels of discrimination and calibration (11).

\section{Development of the Let's Prevent Diabetes programme}

This Let's Prevent Diabetes structured education intervention was developed to meet the need for an evidence based diabetes prevention programme which adheres NICE recommendations and that can be implemented within the NHS.

The Lets Prevent Diabetes intervention encourages self-management of NDH, using simple, nontechnical language and visual aids. The Diabetes Education and Self-Management for On-going and Newly Diagnosed (DESMOND) programme which was the first national education programme for people with T2DM that met NICE criteria was used as a basis for the development of the Let's Prevent Diabetes programme. The development process was informed by the Medical Research Council (MRC) framework. An iterative cycle (including initial development, piloting, collecting and collating qualitative and quantitative data, reflection and modification of the intervention) was used to inform and refine the intervention until it was considered to be fit for purpose for evaluation (12).

The Let's Prevent Diabetes programme is a six hour structured group education session, with three hour refresher sessions at 12 and 24 months after the initial session. These group based sessions were complemented by telephone calls every three months from nursing staff, trained to offer ongoing support in behaviour change and encourage participants in achieving their individual goals. The programme was underpinned by a theoretical basis with a philosophy centred on patient empowerment. The key goals within the curriculum were based on those form the international pivotal trials and included: (1) Sustained weight reduction of $>5 \%$ body weight; (2) Moderate reduction in total fat intake to $<30 \%$ of energy intake; (3) Low saturated fat intake to $<10 \%$ of energy intake; (4) Higher fibre intake of >15 g per 1000 kcals; and (5) Increase step count (all participants are provided with a pedometer).

\section{Cluster randomised trial of the Let's Prevent Diabetes programme}

This Let's Prevent Diabetes programme was evaluated in a cluster randomised trial. Forty four general practices were randomised to receive either standard care $(n=21)$ or the Let's Prevent Diabetes programme $(n=23)$. Within each practice the risk score was used to identify people at high risk of having NDH who were then invited for screening. Of the 17,972 identified as high risk and invited for screening, 3,449 attended (19.2\%). Of those screened, 880 were found to have NDH and were included in the trial (13). Participants either attended the Let's Prevent Diabetes programme or received standard care depending on the allocation of their practice. Participants were followed up for three years and the primary outcome was progression to T2DM (14).

Over the three years, 131 participants developed T2DM (15). There was a $26 \%$ reduced risk of developing T2DM in the intervention arm compared to standard care, but this did not reach statistical significance ( $\mathrm{HR} 0.74,95 \% \mathrm{Cl} 0.48,1.14, \mathrm{p}=0.18)$. The reduction was larger in a per protocol analysis which excluded those from the intervention arm who did not attend the education (HR 0.65 [95\% $\mathrm{Cl} 0.41,1.03])$. There were statistically significant improvements in $\mathrm{HbA1c}$, LDL cholesterol, psychosocial wellbeing, sedentary time and step count in the intervention group compared to the standard care. 
The intervention was found to result in a net gain of 0.046 QALYs over three years at an overall cost of $£ 168$ per patient, with an incremental cost effectiveness ratio of $£ 3,643$ and a probability of $86 \%$ of being cost-effective at a willingness to pay threshold of $£ 20,000$ (16).

\section{Engagement and retention}

Twenty two percent of participants in the intervention arm did not attend the initial six hour session and only $29 \%$ attend the initial session and the two refresher sessions. We conducted a secondary analysis of the trial data to assess if outcome was related to the amount of the intervention received (17). Participants who attended the initial session and at least one refresher session were $62 \%$ less likely to develop T2DM compared to those in the standard care arm (HR 0.38 [95\% $\mathrm{Cl} 0.24,0.62]$ ). This was increased an $88 \%$ reduction in participants who attended all sessions (HR 0.12 [95\% $\mathrm{Cl} 0.05$, $0.28])$.

\section{NHS Diabetes Prevention Programme}

In 2015 the NHS outlined its intention to be the first country to deliver a national evidence-based diabetes prevention programme. The specification for the NHS programme was based on an evidence review of real-world prevention programmes conducted globally which had aimed to translate the findings of the pivotal trials into a routine health care setting (18). This review found that better outcomes were associated with programmes which adhered to NICE guidelines, were delivered over 9-18 months with over 13 sessions, covered both diet and physical activity, used social support, had supervised physical activity, recommended a calorie restricted diet and included 10-15 participants.

In 2016, the NHS commissioned four providers to deliver a National Diabetes Programme. One of the providers (Ingeus UK Ltd) worked in collaboration with the Let's Prevent Diabetes team to adapt the programme to meet the NHS specifications (based on the results of the systematic review). This adapted programme (called Healthier You) will run across 13 shorter sessions over a nine-month period. To date (Dec 2016), the adapted Let's Prevent Diabetes programme is being delivered in Leeds, Leicestershire and South East England (19). Public Health England and NHS England have commissioned evaluations of this initial roll-out. The NIHR have commissioned a larger scale national evaluation.

\section{Conclusions}

We have developed a prevention pathway for T2DM which includes a risk score for use in primary care which can be used as part of a two-stage screening programme to identify those with NDH (See Figure 1). This score is freely available for health care practitioners to use across the UK. Once identified at risk, we have developed a pragmatic cost-effective diabetes prevention programme which leads to improvements in metabolic health, psychological wellbeing and health behaviour over three years, with significant reductions in the progression to T2DM in those who attend the entire programme. An adapted version of this programme is now being delivered nationally by one of the four providers of the NHS Diabetes Prevention Programme. 


\section{Figure 1. Leicester Diabetes Prevention Pathway}

Additional information on each of the aspects is available at http://leicesterdiabetescentre.org.uk/Leicester-Diabetes-Centre-Prevention-Pathway

\section{Leicester Diabetes Centre Prevention Pathway}

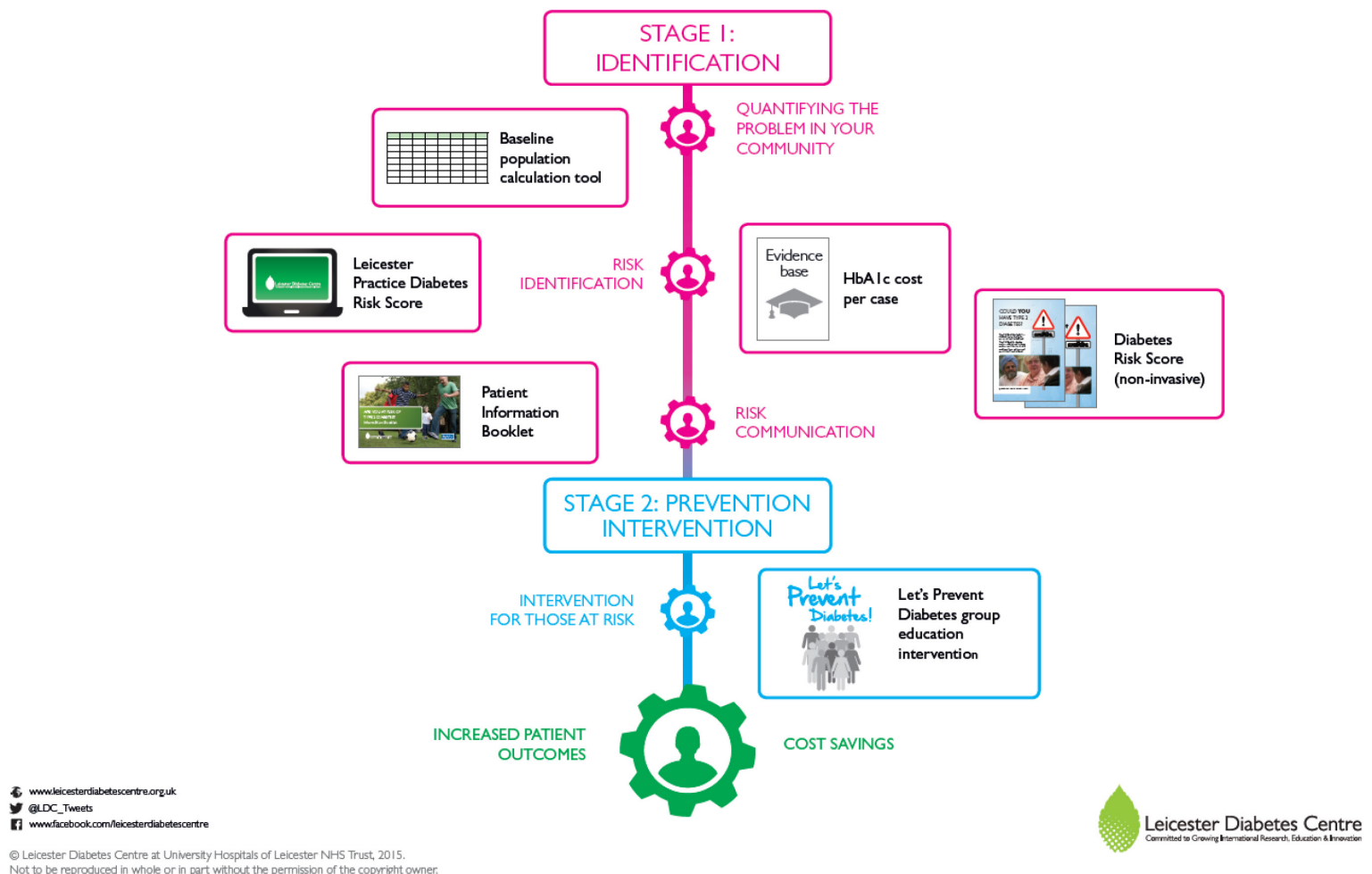




\section{References}

1. NHS. Five year forward view. 2014.

2. Barron E, Gray LJ, Valabhji J, Young B. Prevalence, characteristics, distribution and identification of non-diabetic hyperglycaemia in England. In press. 2016.

3. Gillies CL, Abrams KR, Lambert PC, Cooper NJ, Sutton AJ, Hsu RT, et al. Pharmacological and lifestyle interventions to prevent or delay type 2 diabetes in people with impaired glucose tolerance: systematic review and meta-analysis. BMJ. 2007;334(7588):299.

4. Knowler WC, Barrett-Connor E, Fowler SE, Hamman RF, Lachin JM, Walker EA, et al. Reduction in the Incidence of Type 2 Diabetes with Lifestyle Intervention or Metformin. The New England journal of medicine. 2002;346(6):393-403.

5. Dunkley A, Bodicoat DH, Greaves CJ, Russell C, Yates T, Davies MJ, et al. Diabetes Prevention in the Real World: Effectiveness of Pragmatic Lifestyle Interventions for the Prevention of Type 2 Diabetes and of the Impact of Adherence to Guideline Recommendations. A Systematic Review and Meta-analysis. Diabetes Care. 2014;37:922-33.

6. Chatterton $\mathrm{H}$, Younger T, Fischer A, Khunti K, on behalf of the Programme Development Group. Risk identification and interventions to prevent type 2 diabetes in adults at high risk: summary of NICE guidance. BMJ. 2012;12(345):e4624.

7. Khunti K, Gillies CL, Taub NA, Mostafa SA, Hiles SL, Abrams KR, et al. A comparison of cost per case detected of screening strategies for Type 2 diabetes and impaired glucose regulation: Modelling study. . Diabetes Res Clin Pract. 2012;97(3):505-13.

8. Gray LJ, Taub N, Khunti K, Gardiner E, Hiles S, Webb DR, et al. The Leicester Risk Assessment score for detecting undiagnosed Type 2 diabetes and impaired glucose regulation for use in a multiethnic UK setting. Diabetic Medicine. 2010;27(8):887-95.

9. Gray LJ, Tringham J, Davies MJ, Webb DR, Jarvis J, Skinner TC, et al. Screening for type 2 diabetes in a multiethnic setting using known risk factors to identify those at high risk: a crosssectional study. Journal of Vascular Health and Risk Management. 2010;6:837-42.

10. Webb DR, Khunti K, Srinivasan B, Gray L, Taub N, Campbell S, et al. Rationale and design of the ADDITION-Leicester study, a systematic screening programme and randomised controlled trial of multi-factorial cardiovascular risk intervention in people with type 2 diabetes mellitus detected by screening. Trials. 2010;11:16.

11. Gray LJ, Davies MJ, Hiles S, Taub NA, Webb DR, Srinivasan BT, et al. Detection of impaired glucose regulation and/or type 2 diabetes mellitus, using primary care electronic data, in a multiethnic UK community setting. Diabetologia. 2012;55(4):959-66.

12. Troughton J, Chatterjee S, Hill SE, Daly H, Stacey LM, Stone MA, et al. Development of a lifestyle intervention using the MRC framework for diabetes prevention in people with impaired glucose regulation. Journal of Public Health. 2015:fdv110.

13. Gray LJ, Khunti K, Edwardson C, Goldby S, Henson J, Morris DH, et al. Implementation of the automated Leicester Practice Risk Score in two diabetes prevention trials provides a high yield of people with abnormal glucose tolerance. Diabetologia. 2012;55(12):3238-44.

14. Gray LJ, Khunti K, Williams S, Goldby S, Troughton J, Yates T, et al. Let's Prevent Diabetes: study protocol for a cluster randomised controlled trial of an educational intervention in a multiethnic UK population with screen detected impaired glucose regulation. . Cardiovascular Diabetology. 2012.

15. Davies MJ, Gray LJ, Troughton J, Gray A, Tuomilehto J, Farooqi A, et al. A community based primary prevention programme for type 2 diabetes integrating identification and lifestyle intervention for prevention: the Let's Prevent Diabetes cluster randomised controlled trial. Preventive medicine. 2016;84:48-56.

16. Leal J, Ahrabian D, Gray AM. Cost effectiveness of a pragmatic structured education intervention for type 2 diabetes: economic evaluation of data from the Let's Prevent trial In preparation 2015. 
17. Gray LJ, Yates T, Troughton J, Khunti K, Davies MJ. Engagement, Retention, and Progression to Type 2 Diabetes: A Retrospective Analysis of the Cluster-Randomised" Let's Prevent Diabetes" Trial. PLoS Med. 2016;13(7):e1002078.

18. Ashra N, Spong R, Cater P, Davies MJ, Dunkley A, Gillies CL, et al. A systematic review and meta-analysis assessing the effectiveness of pragmatic lifestyle interventions for the prevention of type 2 diabetes mellitus in routine practice. London 2016.

19. Ingeus UK Ltd. Healthier You - NHS Diabetes Prevention Programme 2016. Available from: http://www.stopdiabetes.co.uk/. 